\title{
EphB6 crosslinking results in costimulation of $T$ cells
}

\author{
Hongyu Luo, ${ }^{1}$ Guang Yu, ${ }^{1}$ Yulian Wu, ${ }^{1,2}$ and Jiangping $\mathrm{Wu}^{1,3}$ \\ ${ }^{1}$ Laboratory of Transplantation Immunology, Centre Hospitalier de l'Universite de Montreal, University of Montreal, \\ Montreal, Canada \\ ${ }^{2}$ Department of Surgery, Second Affiliated Hospital of the Zhejiang Medical College, Zhejiang University, Hangzhou, China \\ ${ }^{3}$ Nephrology Service of Notre Dame Hospital, Centre Hospitalier de l'Universite de Montreal, University of Montreal, \\ Montreal, Canada
}

\begin{abstract}
Erythropoietin-producing hepatocyte (Eph) kinases represent the largest receptor tyrosine kinase family. Some of them are expressed in the $T$ cell compartment, but their function in $T$ cells is unknown. In peripheral blood, EphB6 was predominantly expressed on T cells, and was upregulated after culture. EphB6 crosslinking by anti-EphB6 mAb or ephrinB2 in the presence of suboptimal $\mathrm{T}$ cell receptor (TCR) stimulation led to drastic T cell proliferation, suggesting that EphB6 can costimulate $\mathrm{T}$ cells. The proliferation was accompanied by enhanced production of several lymphokines, such as IFN- $\gamma$, IL-6, IL-10, TGF- $\beta$, TNF- $\alpha$, and GM-CSF, but not IL-2 and IL-4. Sorted EphB6 ${ }^{+} \mathrm{T}$ cells had significantly stronger response to anti-CD3 and anti-CD28 stimulation than EphB6- $T$ cells had. Taken together, these data suggest an important role of EphB6 in normal T cell activation. Within two minutes of anti-CD3 and anti-CD28 stimulation, EphB6 aggregated and colocalized with TCR, and this provides a morphological basis for EphB6 to enhance TCR signaling. The capping was followed by p38 MAPK activation, showing that EphB6 is capable of signaling, in spite of its lack of intrinsic kinase activity. This study demonstrates that interaction between EphB6 and its ligands facilitates $\mathrm{T}$ cell responses to antigen.
\end{abstract}

J. Clin. Invest. 110:1141-1150 (2002). doi:10.1172/JCI200215883.

\section{Introduction}

Protein tyrosine kinases (PTKs) are important enzymes in signal transduction that could be divided into two classes: cytoplasmic and cell-surface PTKs. Most of the latter are important growth factor receptors that are classified as the insulin receptor family, the EGF-R family, the PDGF-R family, the FGF-R family, the nerve growth factor receptor family, the HGF-R family, and the erythropoietin-producing hepatocyte (Eph) kinase family (1). It is obvious from the nomenclature that the immunological significance of most of these receptors is not striking, because they do not have preferential activity or expression in immune cells.

The Eph family is the largest receptor PTK family, comprising about $25 \%$ of known receptor PTKs. They are now named the EphA's (EphA1 to EphA8) and EphB's (EphB1 to EphB6) according to their sequence homology (2). Eph ligands are expressed mainly on cell surfaces and are now termed ephrins. EphrinA's (ephrinA1 to

Received for publication May 8, 2002, and accepted in revised form August 20, 2002

Address correspondence to: Jiangping Wu, Laboratory of Transplantation Immunology, Research Center, Notre Dame Hospital, Centre Hospitalier de l'Universite de Montreal, University of Montreal, Pavilion DeSève, Room Y-5616, 1560 Sherbrooke Street East, Montreal, Quebec H2L 4M1, Canada. Phone: (514) 890-8000 ext. 25164; Fax: (514) 412-7596; E-mail: jianping.wu@umontreal.ca.

Conflict of interest: No conflict of interest has been declared. Nonstandard abbreviations used: protein tyrosine kinase (PTK); erythropoietin-producing hepatocyte (Eph); magnetic cell sorter (MACS); phycoerythrin (PE); phytohemagglutinin (PHA); T cell receptor (TCR).
ephrinA5) are ligands of EphA's and are glycosylphosphatidylinositol-anchored proteins. EphrinB's (ephrinB1 to ephrinB3) are ligands of EphB's and are transmembrane proteins. EphA's bind to ephrinA's and EphB's bind to ephrinBs with loose specificity; generally, EphA's do not bind to the ligands of EphB's, and vice versa. The transmembrane ephrinB's can also function as reciprocal receptors for EphB molecules and transduce signals into cells (3). Since both Eph receptor kinases and their ligands are cell surface molecules, they can interact with each other only if expressed on adjacent cells. Not surprisingly, the clearly demonstrated function of these receptors and ligands is to control accurate spatial patterning and cell positioning. Most of these findings are derived from studies in the CNS, where most Eph kinases have high-level expression (4-10). Recently, it has been found that ephrinB2 and its ligand EphB4 are involved in angiogenesis (10); this function is consistent with the known roles of Eph kinases in controlling spatial structure formation.

Expression in immune cells has not been examined for many members of the Eph family, but only a few of those that have been studied are expressed in immune cells. EphA3 is expressed in pre-B cell lines (11). EphA2 (12), EphB4 (13), and EphB6 (14) are expressed at high levels in the thymus. The function of this important family of PTKs in the immune system has not been explored, to the best of our knowledge, with the exception of our recent publication on EphB6 (15). However, Shimoyama et al. have recently reported the expression pattern of EphB6 in hematopoietic cells (16). 
EphB6 shares about 47-60\% homology with other members of the EphB subgroup, but the homology between human and mouse EphB6 is more than 90\% $(14,17)$. This high degree of homology suggests an important conserved function of EphB6, although it lacks intrinsic tyrosine kinase activity due to a mutation in its kinase domain $(14,17)$. EphB6 is expressed at high levels in the brain and thymus $(14,17)$ and in Jurkat cells, a human $\mathrm{T}$ cell leukemia line (15). Its mRNA is detectable in normal T cells and B cells, with the former having much higher expression (15). Our recent work showed that crosslinking EphB6 on Jurkat cells leads to increased secretion of GM-CSF accompanied by Fas-mediated apoptosis (15). Crosslinking EphB6 augments protein tyrosine phosphorylation, indicating that signals can indeed be transduced into Jurkat cells through EphB6. EphB6 signaling is likely achieved by its association with a number of adaptor proteins, such as $\mathrm{Cbl}, \mathrm{Grb} 2$, and $\mathrm{CrkL}$, that are involved in signaling pathways (15).

In this study we examined expression of EphB6 in immune cells and explored its function and signaling in normal $\mathrm{T}$ cells.

\section{Methods}

T cell purification and culture. Human PBMCs were isolated by Lympholyte gradient (Cedarlane Laboratories Ltd., Hornby, Ontario, Canada) as described elsewhere (18). T cells were purified from PBMCs by negative selection (deletion of cells positive for CD11b, CD16, CD19, CD36, and CD56) with a magnetic cell sorter (MACS; Miltenyi Biotech, Auburn, California, USA) according to the manufacturer's instructions. The purified cells were more than 98\% CD3-positive. In some experiments, these cells were further selected by anti-CD4, anti-CD8, and anti-CD45RO magnetic beads. In some other experiments, these $T$ cells were cultured in medium for 48 hours in order to enhance EphB6 expression. These cells were then stained with biotin-conjugated anti-EphB $6 \mathrm{mAb}$ followed by phycoerythrin-conjugated (PE-conjugated) streptavidin, and then sorted by high-speed flow cytometry. Cells with the highest $30 \%$ and lowest 30\% fluorescence intensity were collected and designated as $\mathrm{EphB6}{ }^{+}$and EphB6- cells, respectively. These cells were more than $98 \%$ CD3-positive, and were less than $2 \%$ propidium iodide- and annexin $\mathrm{V}$-positive, indicating high purity and viability. In some experiments, $T$ cells were cultured in wells coated with anti-CD3 $\mathrm{mAb}$ (clone OKT3), anti-EphB6 mAb (clone 4F12; ref. 15), antiCD28, or mouse ephrinB2-Fc. The cells were cultured in RPMI 1640 supplemented with 10\% FCS, L-glutamine, and antibiotics. ${ }^{3} \mathrm{H}$-thymidine uptake was measured as described previously (18).

Generation of human EphB6-Fc and mouse ephrinB2-Fc. The coding sequences of the extracellular domains of human EphB6 from positions 799 to 2,442 (GenBank accession number NM004445) and mouse ephrinB2 from positions 29 to 684 (GenBank accession number NM010111) were cloned in-frame upstream of the human $\operatorname{IgG}_{1} \mathrm{Fc}$ coding sequence in expression vector $\mathrm{pCMVh-Fc}$. The constructs and pcDNA3 were then transfected into $\mathrm{CHO} / \mathrm{dhfr}^{-}$cells with Lipofectamine (Invitrogen, Burlington, Ontario, Canada). The cells were cultured in selection medium, which was $\alpha$-MEM without ribonucleosides and deoxyribonucleosides, but with 5\% dialyzed FCS, 0.01 $\mathrm{mM}$ methotrexate, $0.8 \mathrm{mg} / \mathrm{ml} \mathrm{G} 418$, and $0.1 \mathrm{mg} / \mathrm{ml}$ gentamicin. After 2 weeks of culture, well-isolated clones were handpicked and expanded in the selection medium without G418. The culture supernatants were assayed by ELISA for human IgG-Fcpositive clones, which were then expanded. The fusion proteins were isolated from their supernatants by protein A columns, analyzed with $12 \%$ SDS-PAGE to confirm their molecular sizes, and verified by $\mathrm{N}$-terminal peptide sequencing.

Flow cytometry. Two- or three-color flow cytometry was employed for measurement of EphB6 expression in different cell populations. Cells were labeled with biotinylated anti-EphB6 mAb 4F12 followed by PE-conjugated streptavidin (Sigma-Aldrich, Oakville, Ontario, Canada) in combination with FITC-labeled anti-CD4 (clone SFCI12T4D11; Beckman Coulter Inc., Burlington, Ontario, Canada), FITC-labeled antiCD8 (clone UCHT-4; Sigma-Aldrich), Quantum Red-labeled anti-CD45RO (clone UCHL-1; SigmaAldrich), FITC-labeled anti-CD45RA (clone F8-11-13; Sigma-Aldrich), FITC-labeled anti-CD20 (clone HI147; Caltag Laboratories Inc., Burlingame, California, USA), or FITC-labeled anti-CD14 (clone MO2; Beckman Coulter Inc.). Activation markers and an adhesion molecule on $\mathrm{T}$ cells were detected by onecolor flow cytometry on MACS-purified T cells, using FITC-labeled anti-CD25 (clone M-A251), FITC-labeled anti-CD69 (clone FN50), or PE-labeled anti-CD54 (clone HA58). These mAbs were from Pharmingen (San Diego, California, USA).

Cytokine measurement. Culture supernatants of $\mathrm{T}$ cells placed in anti-CD3- and/or anti-EphB6-coated wells were harvested 48 hours after initiation of culture. IL-2, IL-4, IL-6, IL-10, IFN- $\gamma$, TNF- $\alpha$, TGF- $\beta$, and GM-CSF in the supernatants were quantified by ELISA (Quantikine; R\&D Systems Inc., Minneapolis, Minnesota, USA) according to the manufacturer's instructions.

Laser scanning confocal microscopy. MACS-purified T cells were cultured for 48 hours. The cells were incubated on ice for 40 minutes with Alexa Fluor 488 -conjugated anti-CD3 mAb $\left(0.5 \mu \mathrm{g} / 10^{6}\right.$ cells $)$ (Molecular Probes Inc., Eugene, Oregon, USA) and anti-CD28 mAb ( $1 \mu \mathrm{g} / 10^{6}$ cells). After washing, the cells were reacted with rabbit anti-mouse IgG (1 $\mu \mathrm{g} / 10^{6}$ cells) at $37^{\circ} \mathrm{C}$ for 2 minutes. Cells were immediately fixed with $4 \%$ paraformaldehyde on ice for 30 minutes and then stained with biotin-conjugated anti-EphB6 mAb (clone 4F12; ref. 15) or biotin-conjugated anti-CD45 (clone HI30; Caltag Laboratories Inc., Burlingame, California, USA), at $1 \mu \mathrm{g} / 10^{6}$ cells, 
followed by Alexa Fluor 594-conjugated streptavidin $\left(1 \mu \mathrm{g} / 10^{6}\right.$ cells $)$. The cells were then examined by laser-scanning confocal microscopy.

Immunoblotting. T cells were added to 6-well Nunc plates (Nalge Nunc International, Naperville, Illinois, USA) coated with anti-EphB $6 \mathrm{mAb}$, anti-CD3 mAb, or both. The cells were harvested after 4 hours or 20 hours in culture, and were lysed with $1 \%$ NP-40 lysis buffer. Cleared lysates were resolved using 10\% SDS-PAGE with $50 \mu \mathrm{g}$ protein per lane, and were then blotted onto PVDF membranes. The membranes were sequentially hybridized with rabbit $\mathrm{Ab}$ against tyrosine phosphorylated p38 MAPK and rabbit anti-p38 MAPK Ab (Cell Signaling Technology Inc., Beverly, Massachusetts, USA). Signals were revealed by enhanced chemiluminescence. Details of the immunoblotting method have been provided in our previous publication (19).

\section{Results}

EphB6 expression in immune cells measured by flow cytometry. We examined EphB6 expression on the immune cell surface. $\mathrm{CD}^{+}$and $\mathrm{CD}^{+}$cells were double-labeled with anti-CD4/anti-EphB6 or anti-CD8/anti-EphB6 and analyzed by flow cytometry. Cells having EphB6 intensity above the $\mathrm{mAb}$ isotypic control were designated EphB6-positive cells, which were not a separate population but a continuum extended from EphB6negative cells. In freshly prepared cells, $12.6 \% \mathrm{CD}^{+}$ cells and $10.6 \% \mathrm{CD}^{+}$cells were EphB6 positive (Figure 1a). More detailed study revealed that $\mathrm{CD} 4^{+}$and $\mathrm{CD}^{+}$cells rapidly upregulated their EphB6 after culture in medium. For $\mathrm{CD}^{+}$cells, the upregulation was significant within 4 hours, when $39.3 \%$ were found to be EphB6 ${ }^{+}$(Figure 1a). At 24 hours, 52.9\% were positive, and this high expression lasted for at least 72 hours. The upregulation was reflected not only in the percentage increase of cells in the EphB6-positive region, but also in the increase of EphB6 expression intensity of the whole $\mathrm{CD} 4^{+}$population, which was shifted to the right in histograms. For $\mathrm{CD}^{+}$cells, the upregulation was similar in scale and duration to that of $\mathrm{CD}^{+}$cells, but occurred at a slower pace; it became significant only at 24 hours. The upregulation was not due to stimulatory factors in FCS, since $\mathrm{T}$ cells cultured in serum-free medium also upregulated their EphB6 (Figure 1b). Interestingly, T cells cultured in fresh autologous serum failed to significantly upregulate their EphB6 (Figure 1b). This suggests that certain serum components prevent EphB6 upregulation on T cells; this phenomenon probably has physiological significance (see Discussion). We further determined that constitutive as well as upregulated EphB6 expression in vitro depended on de novo protein and mRNA synthesis. As shown in Figure 1c, inhibition of protein and mRNA synthesis by cycloheximide and actinomycin D, respectively, for 4 hours started to prevent EphB6 upregulation; at 24 hours, they both totally eliminated EphB6 expression on $\mathrm{CD}^{+}$and $\mathrm{CD}^{+}$cells. This implies that the preexisting EphB6
mRNA or protein has a turnover rate of less than 24 hours, or else the mRNA or protein will maintain EphB6 expression during this period in the presence of mRNA and protein synthesis inhibitors.

Interestingly, mitogen (phytohemagglutinin; PHA) activation marginally increased EphB6 expression in $\mathrm{CD}^{+}$and $\mathrm{CD}^{+}$cells at 4 hours compared with cells without stimulation $(39.3 \%$ in unstimulated versus $49.8 \%$ in PHA-stimulated $\mathrm{CD}^{+}$cells; $13.9 \%$ in unstimulated versus $16.4 \%$ in PHA-stimulated $\mathrm{CD}^{+}$cells), but downregulated EphB6 expression at 24 hours to levels below those of fresh cells, with only $7.8 \%$ and $5.3 \%$ EphB6 $6^{+} \mathrm{CD}^{+}$and $\mathrm{CD} 8^{+}$cells, respectively (see Figure 1c). Again, the mitogen-induced increase followed by a decrease was reflected not only in changes of EphB6 ${ }^{+}$ percentages, but also in the fluorescent intensity of the whole population. The downregulation of EphB6 expression on the cell surface after mitogen stimulation was consistent with our previous finding that EphB6 mRNA was reduced after T cell activation (15). Such downregulation could be a mechanism for $T$ cells to terminate EphB6 signaling after their activation.

We also examined EphB6 expression on T cells that had experienced activation $\left(\mathrm{CD} 45 \mathrm{RO}^{+}\right)$or those having a naive phenotype $\left(\mathrm{CD}^{2} 5 \mathrm{RA}^{+}\right)$. As shown in Figure 1d, both types of T cells expressed EphB6 after 24 hours in culture, although CD45RA ${ }^{+}$cells had higher expression

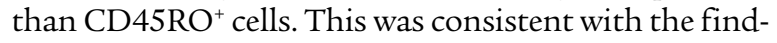
ing that $\mathrm{T}$ cell activation led to downregulation of EphB6 expression.

EphB6 expression in freshly isolated B cells and monocytes/macrophages was very limited, with only $1.9 \%$ EphB $6^{+}$cells in the $\mathrm{CD} 20^{+}$population, and $3.4 \%$ EphB6 $6^{+}$cells in the $\mathrm{CD}_{14}{ }^{+}$population (Figure 1e). Unlike T cells, B cells or monocytes/macrophages cultured in medium did not have enhanced EphB6 expression (data not shown). Therefore, in the immune system, EphB6 was expressed predominantly on T cells, and consequently, its major role in the immune system should be related to $T$ cell function.

Crosslinking EphB6 enhanced the T cell response to T cell receptor stimulation. In a recent publication, we reported that EphB6 and suboptimal anti-CD3 co-crosslinking resulted in Jurkat cell apoptosis (15). It is known that with strong (not with suboptimal) $T$ cell receptor (TCR) stimulation, Jurkat cells undergo apoptosis. We speculated that EphB6 crosslinking enhanced signaling strength through TCR and increased TCR stimulation from suboptimal to strong, which led to Jurkat cell apoptosis. We therefore reasoned that EphB6 crosslinking should enhance the activation of normal resting $\mathrm{T}$ cells triggered by TCR stimulation. Testing of this possibility showed that suboptimal anti-CD3 or antiEphB6 alone caused negligible T cell proliferation (Figure 2a), but anti-EphB6 dose-dependently induced proliferation in the presence of anti-CD3. Next, T cells were cultured in wells coated with an optimal amount of anti-EphB6 and various amounts of anti-CD3. As shown in Figure 2b, anti-EphB6 augmented $\mathrm{T}$ cell 


\section{Figure 1}

Flow cytometry analysis of EphB6 expression on immune cells. (a) MACS-purified $\mathrm{CD}^{+}$or $\mathrm{CD}^{+} \mathrm{T}$ cells (more than $98 \%$ pure) were cultured for different times and analyzed for EphB6 expression by two-color flow cytometry using FITC-labeled anti-CD4 or FITClabeled anti-CD8 in combination with biotinconjugated anti-EphB6 and PE-streptavidin. Histograms show EphB6 expression on cells gated on $\mathrm{CD}^{+}$or $\mathrm{CD} 8^{+}$populations. Percentages represent $\mathrm{EphB6}^{+}$populations in the gated regions after deducting background staining (isotypic $A b$, shaded area); this is also the case for all other histograms in Figure 1. (b) MACS-purified T cells were cultured in regular RPMI 1640 medium, 100\% fresh serum from the blood donor (autologous serum), or serum-free medium for 24 hours. EphB6 expression was examined by one-color flow cytometry. (c) MACS-purified $\mathrm{CD} 4^{+}$or $\mathrm{CD} 8^{+}$ cells were cultured in the presence of $\mathrm{PHA}$ ( 1 $\mu \mathrm{g} / \mathrm{ml})$, actinomycin $\mathrm{D}(\mathrm{AD} ; 4 \mu \mathrm{g} / \mathrm{ml})$, cycloheximide ( $\mathrm{CHX} ; 20 \mu \mathrm{g} / \mathrm{ml}$ ), or in medium (Med) alone for the indicated time periods. Cells were stained and analyzed as described in a. (d) MACS-purified T cells were cultured for 24 hours and then stained with Quantum Red-labeled anti-CD45RO, FITC-labeled antiCD45RA, and biotin-conjugated anti-EphB6 followed by PE-streptavidin. The CD45RO ${ }^{+}$ and CD45RA ${ }^{+}$cells were separately gated, and their EphB6 expression was assessed. (e) The T cell-depleted fraction of PBMCs was stained with FITC-labeled anti-CD20 or FITC-labeled anti-CD14 in combination with biotin-conjugated anti-EphB6 and PE-streptavidin, and analyzed by two-color flow cytometry. EphB6 expression on cells gated on $\mathrm{CD} 20^{+}$or CD14 populations is shown.
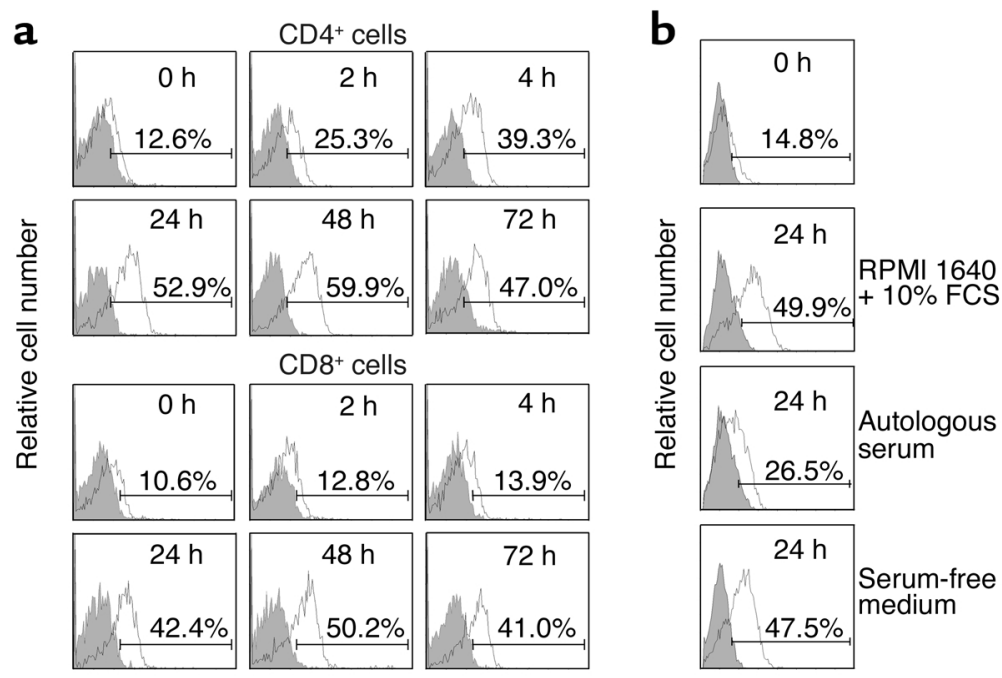

EphB6 log fluorescence intensity

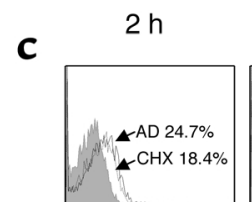

$4 \mathrm{~h}$

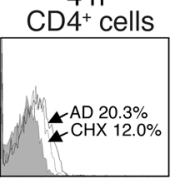

$24 \mathrm{~h}$

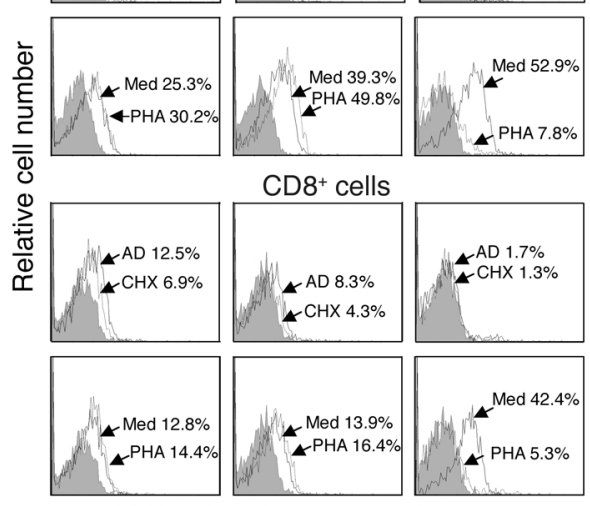

EphB6 log fluorescence intensity
EphB6 log fluorescence intensity

d
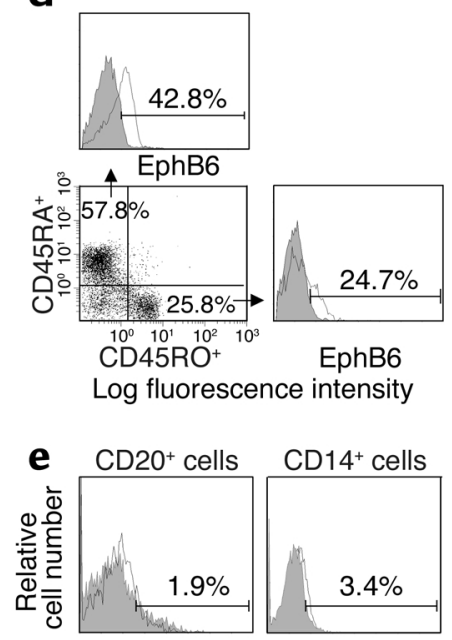

EphB6 log fluorescence intensity proliferation when anti-CD3 was used, at all concentrations tested. This result showed that EphB6 crosslinking reduced the $\mathrm{T}$ cell response threshold. Therefore, in a broad sense, EphB6 could be considered a novel $\mathrm{T}$ cell costimulatory molecule.

To show the specificity of anti-EphB6 stimulation, recombinant EphB6-Fc was added to the culture. As shown in Figure 2c, soluble EphB6-Fc, but not a control fusion protein with an Fc tail, reduced solid-phase antiCD3 plus anti-EphB6-stimulated T cell proliferation.

We further demonstrated that $\mathrm{CD}^{+}$cells and $\mathrm{CD}^{+}$ cells responded similarly to EphB6 costimulation, although $\mathrm{CD}^{+}$cells had a tendency to reach their proliferation peak later than $\mathrm{CD} 4^{+}$cells (Figure 2d). This was in keeping with the slower EphB6 upregulation in $\mathrm{CD}^{+}$cells shown in Figure 1a. The magnitude of costimulation by EphB6 was slightly lower than, but on the same order of, that using the classical costimulating molecule CD28 (Figure 2d). We also showed that both $\mathrm{CD}^{2} 5 \mathrm{RO}^{+}$and $\mathrm{CD}^{2} 5 \mathrm{RO}^{-} \mathrm{T}$ cells proliferated well upon EphB6 costimulation (Figure 2e).
To show the physiological relevance of EphB6 in T cell activation, we used ephrinB2, the natural ligand of EphB6 (20), for the crosslinking. As we have not yet generated human recombinant ephrinB2, mouse ephrinB2 was used instead. (Mouse ephrinB2 shares 95\% and $97 \%$ homology with human ephrinB2 in its extracellular domain at the mRNA and protein levels, respectively.) $\mathrm{T}$ cells were cultured on solid-phase mouse ephrinB2 along with a suboptimal amount of anti-CD3. As shown in Figure 2f, T cells proliferated vigorously upon stimulation by solid-phase ephrinB2 in the presence of a suboptimal concentration of solid-phase anti-CD3. Thus, the natural ligand of EphB6, as with anti-EphB6 mAb, could costimulate T cells in the presence of TCR ligation. It is acknowledged that since ephrinB2 could bind to other EphB family members, notably EphB4, which is expressed in lymphoid cells (14), the stimulatory effect of ephrinB2 in this experiment might be mediated by more than one receptor. Nevertheless, considering both the specificity of the anti-EphB6 mAb and the effect of 
EphB6's natural ligand ephrinB2, we have shown that EphB6 crosslinking can costimulate T cells.

EphB6 costimulation led to upregulation of activation-related surface markers and lymphokine secretion by T cells. Flow cytometry was used to analyze cell-surface molecules 24 hours after EphB6 and CD3 co-crosslinking (Figure 3a). IL-2 receptor CD25, adhesion molecule CD54, and $\mathrm{T}$ cell activation marker CD69 were drastically upregulated. In all these cases, crosslinking CD3 (a suboptimal amount) or EphB6 alone had no apparent effect. Thus, $T$ cells showed typical activation surface marker expression with CD3 and EphB6 co-crosslinking; this was consistent with their enhanced proliferation, as described in the previous section.

We next assessed the functional consequence of $\mathrm{T}$ cell activation by EphB6 costimulation in terms of lymphokine production. $\mathrm{T}$ cells stimulated with solidphase anti-EphB6 or suboptimal anti-CD3 alone had basal levels of eight tested lymphokines (Figure 3b). When the two stimulations were combined, there were ten- to 100 -fold increases in IFN- $\gamma$, TGF- $\beta$, TNF- $\alpha$, and GM-CSF secretion, and a two to threefold rise in IL-10 and IL- 6 production after 48 hours. On the other hand, secretion of IL-2 and IL-4 was not induced on day 2 . These were not detectable on day 1 or day 3 (data not shown). This excluded the possibility that the failure of detection of these cytokines was due to consumption or due to a shift in secretion kinetics.

EphB6 costimulation occurred among T cells. If EphB6 is important in $\mathrm{T}$ cell activation, what will happen if $\mathrm{T}$ cells are EphB6 negative? To answer this question, we cultured $T$ cells for 2 days to allow upregulation of their EphB6, and then sorted the cells according to their EphB6 expression. T cells with the highest 30\% and lowest 30\% EphB6 fluorescence intensity were defined as EphB6 ${ }^{+}$or EphB6- populations, respectively a

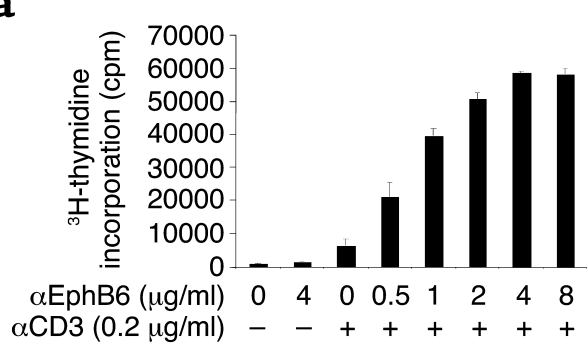

b

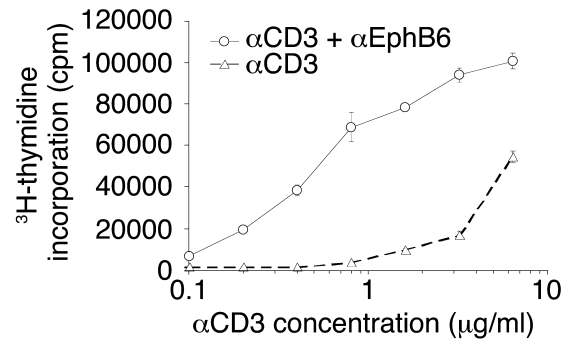

c
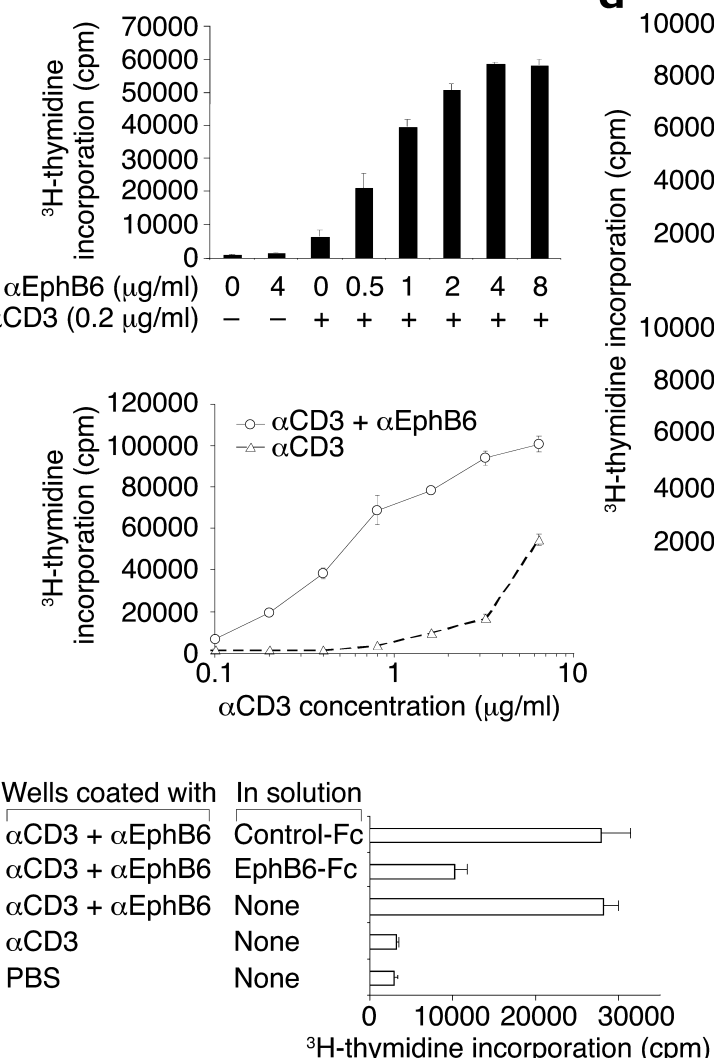

d

\section{f}

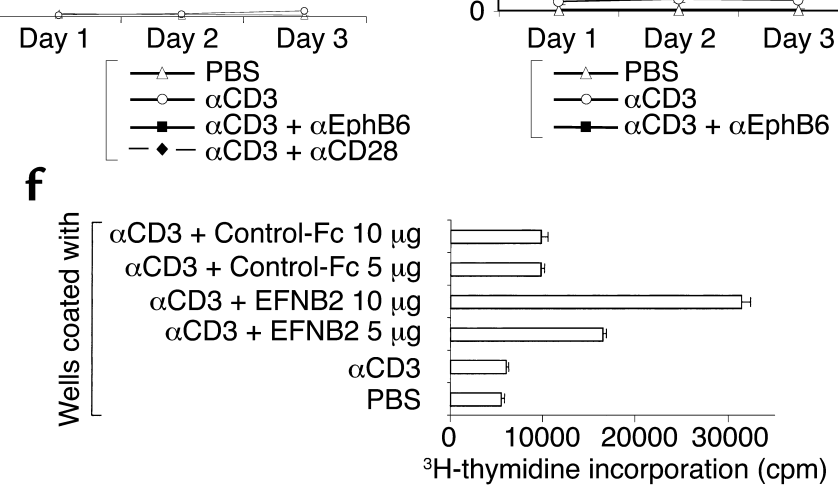

\section{Figure 2}

EphB6 crosslinking enhances the T cell response to TCR stimulation. Representative results are shown. (a and b) MaxiSorp 96-well plates were coated overnight at $4{ }^{\circ} \mathrm{C}$ with a suboptimal concentration of $\alpha \mathrm{CD} 3 \mathrm{mAb}$ (clone OKT3, $0.2 \mu \mathrm{g} / \mathrm{ml}$ ) along with various concentrations of $\alpha$ EphB6 mAb (clone 4F12) (a), or were coated with an optimal concentration of $\alpha$ EphB6 $(4 \mu \mathrm{g} / \mathrm{ml})$ along with various concentrations of $\alpha C D 3(\mathbf{b})$. MACS-purified T cells were cultured in these plates for 72 hours, and ${ }^{3} \mathrm{H}$-thymidine was added for the last 8 hours of culture. (c) MASC-purified T cells were cultured in these wells in the presence or absence of soluble EphB6-Fc or a control protein (control-Fc) (10 $\mu \mathrm{g} / \mathrm{ml}$ ) as inhibitor. (d and $\mathbf{e}$ ) MaxiSorp 96-well plates were coated with $\alpha$-CD3 plus $\alpha$-EphB6, or with $\alpha$-CD3 plus $\alpha$ CD28 (4 $\mu \mathrm{g} / \mathrm{ml}$ ) as described above. T cells were purified from PBMCs by MACS and subsequently fractionated into $\mathrm{CD} 4^{+}$cells, $\mathrm{CD} 8^{+}$cells, CD45RO ${ }^{+}$cells, and $\mathrm{CD}^{2} 5 \mathrm{RO}^{-}$cells (pure T cells depleted of $\mathrm{CD}^{2} 5 \mathrm{RO}^{+}$cells) using MACS beads. The cells were cultured for 1-3 days, and their thymidine uptake was measured for the last 8 hours of culture. (f) Costar 96 -well plates were first coated with $\alpha-C D 3(0.2 \mu \mathrm{g} / \mathrm{ml})$ overnight. After washing, the wells were incubated with mouse ephrinB2-Fc or a control recombinant protein with an $\mathrm{Fc}$ tag (control-Fc) $(5 \mu \mathrm{g} / \mathrm{ml} \mathrm{or} 10 \mu \mathrm{g} / \mathrm{ml}$, as indicated) for 2 hours at $37^{\circ} \mathrm{C}$ followed by 2 hours on ice. MACS-purified T cells were cultured in the wells for 48 hours, and thymidine uptake was measured for the last 8 hours of culture. $\alpha$, anti. 

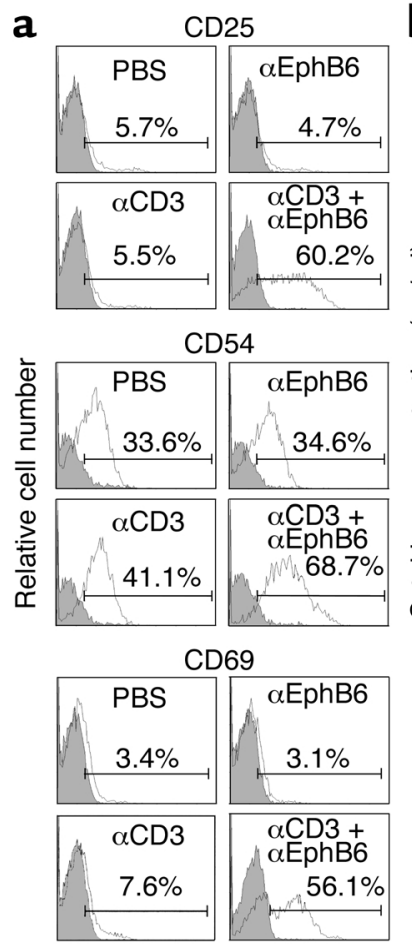

Log fluorescence intensity b
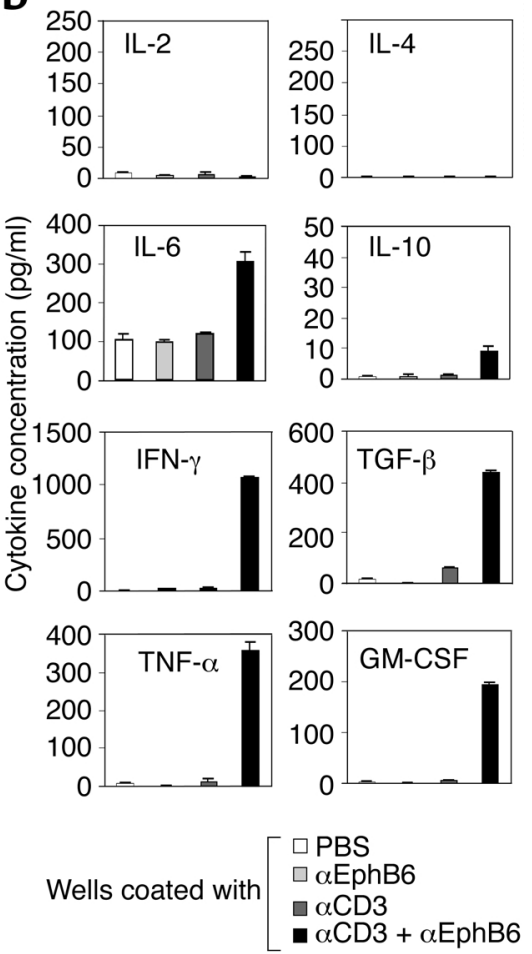

\section{Figure 3}

Activation marker expression and lymphokine secretion of EphB6-costimulated T cells. All experiments were carried out more than three times and were reproducible. Representative results are shown. (a) MACS-purified T cells were cultured in wells coated with anti-EphB6 mAb $(4 \mu \mathrm{g} / \mathrm{ml})$ plus anti-CD3 mAb $(0.2 \mu \mathrm{g} / \mathrm{ml})$ as described in Figure 2. Their CD25, CD54, and CD69 expression at 24 hours was assessed by one-color flow cytometry using FITC-labeled anti-CD25, PElabeled anti-CD54, and FITC-labeled anti-CD69. Percentages represent positive populations in the gated regions after deducting background staining (isotypic $A b$, shaded area). (b) MACS-purified T cells were cultured in wells coated with anti-EphB6 mAb $(4 \mu \mathrm{g} / \mathrm{ml})$, anti-CD3 $\mathrm{mAb}(0.2 \mu \mathrm{g} / \mathrm{ml})$, or both, for 48 hours. IL-2, IL-4, IL- 6 , IL-10, IFN- $\gamma$, TGF- $\beta$, TNF- $\alpha$ and GM-CSF in supernatants were measured by ELISA, with samples in duplicate. Data are expressed as mean \pm SD.

(Figure 4a). These two populations were both more than $98 \%$ CD3-positive, and less than $2 \%$ were positive for propidium iodide and annexin $\mathrm{V}$ (data not shown), indicating high purity and viability of the sorted cells. The cells were stimulated with a suboptimal amount of solid-phase anti-CD3 plus an optimal amount of antiCD28. After 24 hours, cell surface activation markers were examined by flow cytometry. As shown in Figure $4 \mathrm{a}, \mathrm{EphB6}^{+}$cells had higher CD25 expression than EphB6- cells when stimulated with anti-CD3 plus antiCD28 (69.9\% vs. 46.8\%). For CD69 expression, almost all cells in both populations became CD69-positive (last panel, region A, 97.9\% vs. 98.6\%), indicating that $\mathrm{EphB6}^{+}$ and EphB6 ${ }^{-}$cells were viable and activable. However, 95.3\% of the EphB6 $6^{+}$cells expressed high-intensity CD69 (region B), while only $58.1 \%$ of the EphB6- cells did so. The rest of the EphB6- cells expressed CD69 at intermediate intensity. On the other hand, when stimulated with anti-CD3 alone, EphB6- cells were better than EphB6 ${ }^{+}$cells at upregulating CD25 (21.4\% vs.
$10.9 \%$ ) and CD69 (35.2\% vs. $6.9 \%)$. The underlying mechanism of this difference is not clear at this time.

EphB6 ${ }^{+}$and EphB6- $\mathrm{T}$ cells were significantly different in terms of proliferation, as shown in Figure 4b. Upon stimulation with solid-phase anti-CD3 and anti-CD28, $\mathrm{EphB}^{+}$cells had vigorous proliferation, whereas EphB6- cells did not. Since there were only $\mathrm{T}$ cells in this system, the enhanced response in EphB6 ${ }^{+} \mathrm{T}$ cells must be due to costimulation of their EphB6 by their ligands on other T cells. Thus, EphB6 was involved in cooperation among $\mathrm{T}$ cells for optimal T cell activation.

EphB6 signaling. To understand how EphB6 provided costimulation to T cells, we first examined the localization of EphB6 on the T cell surface by confocal microscopy. In resting T cells, EphB6 was distributed in a diffused fashion on the cell surface (Figure 5a). When the T cells were activated by anti-CD3 and anti-CD28 crosslinking, the TCR complex, which was stained green by Alexa Fluor 488-conjugated anti-CD3, formed caps within 2 minutes, as expected. Interestingly, within this time frame, EphB6 (which was stained red by biotin-conjugated anti-EphB6 followed by Alexa Fluor 594-conjugated streptavidin) congregated and colocalized with the TCR caps. This provided a physical basis for EphB6 to interact with the TCR signaling machinery in the caps and to enhance the TCR signaling. The co-capping of $\mathrm{CD} 3$ and EphB6 in this experiment was not due to nonspecific trapping of EphB6 during anti-CD3 and anti-CD28 crosslinking, since the same procedure did not cause another cell-surface molecule, CD45, to cocap with CD3 (Figure 5b).

EphB6 has no intrinsic kinase activity but is associated with several adaptors such as $\mathrm{Cbl}, \mathrm{Grb} 2$, and $\mathrm{CrkL}$ (15). It probably uses these to interact with other signaling pathways. We examined the activation of p38 MAPK, a downstream signaling molecule in the Ras pathway, after EphB6 crosslinking. As shown in Figure $5 c$, a combination of EphB6 and suboptimal CD3 crosslinking led to a long-lasting increase of p38 MAPK phosphorylation from 4 hours to 20 hours, while these two crosslinkings had little effect independently, according to immunoblotting. The membrane was reprobed with anti-p38 MAPK Ab, and $\mathrm{p} 38$ protein levels showed no apparent change. Activation of p38 MAPK in this case was an essential and relevant event in T cell activation after EphB6 and CD3 co-crosslinking, since the p38 MAPK-specific inhibitor SB203580 but not its nonfunctional analogue SB202474 strongly suppressed $\mathrm{T}$ cell proliferation (Figure $5 \mathrm{~d}$ ). 


\section{Discussion}

In this study, we report for the first time to our knowledge on the biological functions of an Eph kinase in normal human $T$ cells. Eph kinases represent the largest family of receptor tyrosine kinases, and several of them, including EphB6, have high mRNA expression in the T cell compartment of the immune system. It is surprising that up to now, the expression and function of EphB6 in the immune system has evaded our notice, despite many successful attempts in generating Abs against $T$ cell surface proteins using either fresh or activated $\mathrm{T}$ cells as immunogens. We think the major reason for this is that EphB6 has low expression on freshly isolated blood (Figure 1), and $\mathrm{T}$ cell activation further reduces its expression. The drastic increase of EphB6 expression from $10 \%$ to about $50 \% \mathrm{~T}$ cells after culture is interesting. There are several possible mechanisms for low EphB6 expression on freshly isolated $\mathrm{T}$ cells and its subsequent upregulation in culture: (a) EphB6, like some other receptor kinases, is downregulated after encountering its ligand(s) in vivo. This is a distinct possibility, because its known ligand, ephrinB2, is expressed on the endothelium of blood vessels (10). Moreover, the fact that pure EphB6 $6^{+} \mathrm{T}$ cells responded much better than EphB6- T cells to TCR stimulation suggested that EphB6 ligand(s) is expressed on the cell surface as well. It is plausible that in vivo, when $\mathrm{T}$ cells are closely packed in lymphoid organs or have frequent contact with endothelial cells, EphB6 and its ligand(s) are constantly engaged, and EphB6 is consequently downregulated. (b) EphB6 expression on T cells is suppressed by serum factors. (c) Serum proteases constantly cleave EphB6 from the T cell surface. The evidence favoring the latter two mechanisms is that when $T$ cells were cultured in serum-free medium, they rapidly upregulated their EphB6 expression, while fresh autologous serum prevented it. All these possibilities are currently under further investigation.

Constitutive and upregulated EphB6 expression on $\mathrm{T}$ cells depended on constant de novo protein and mRNA synthesis, since blocking these two processes for 24 hours resulted in total disappearance of EphB6 expression. Why does the immune system waste energy constantly making new EphB6 but at the same time trying to eliminate its surface expression? There are three interesting observations related to the answer of this question: (a) EphB6 reduced the response thresholds to concomitant suboptimal TCR stimulation in resting T cells; (b) T cells precrosslinked by anti-EphB6 also strongly responded to subsequent suboptimal TCR crosslinking (data not shown); and (c) EphB6 on T cells probably has intimate and constant contact with its ligands in vivo, as explained above. A combination of these three events makes $\mathrm{T}$ cells in lymphoid organs ever ready to respond with low thresholds to antigen. This is likely one of the reasons why immune responses take place preferentially in these organs. Continuous emergence of new EphB6 molecules allows signals to be transduced through them, while old EphB6 is eliminated because the signaling machinery it associates with is exhausted due to constant receptor engaging. The energy wasted in EphB6 synthesis and elimination might well be compensated for by energy saved in decreasing the $\mathrm{T}$ cell response threshold and by the advantage gained in having an ever-ready highly responsive T cell population. Further study is necessary to prove this theory.

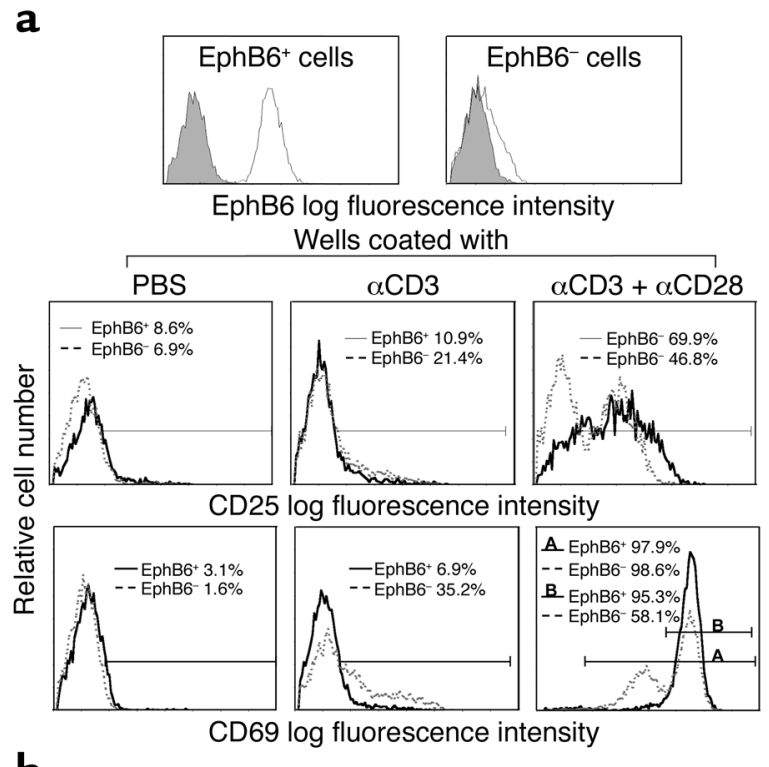

b

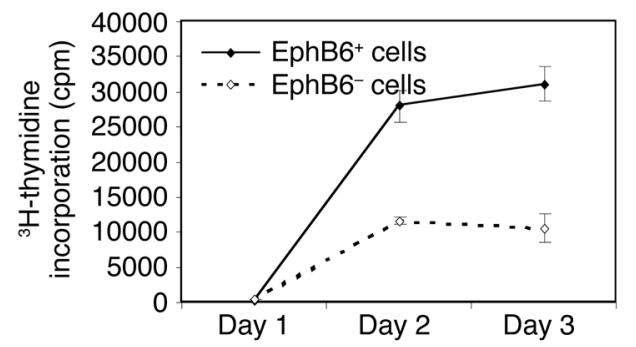

\section{Figure 4}

Activation marker expression and proliferation of EphB6 ${ }^{+}$versus EphB6- T cells. All experiments were performed more than three times and were reproducible. Representative results are shown. T cells were first purified from PBMCs with negative selection using MACS beads. The $T$ cells were cultured in medium for 48 hours to achieve high EphB6 expression, and then stained with biotin-conjugated antiEphB6 mAb followed by PE-streptavidin. The cells with the highest $30 \%$ and lowest 30\% EphB6 fluorescence intensity were sorted by high-speed flow cytometry. (a) The top row shows EphB6 expression of sorted EphB6 ${ }^{+}$and EphB6- T cells. The lower two rows show CD25 and CD69 expression after stimulation with solid-phase anti-CD3 $\mathrm{mAb}$ alone $(0.2 \mu \mathrm{g} / \mathrm{ml}$ during coating) or with anti-CD3 plus anti$\mathrm{CD} 28 \mathrm{mAb}(4 \mu \mathrm{g} / \mathrm{ml}$ during coating) for 24 hours after sorting. Solid lines represent EphB6 ${ }^{+} \mathrm{T}$ cells and dotted lines represent EphB6- $\mathrm{T}$ cells. The percentages represent cells in $\mathrm{CD} 25^{+}$or $\mathrm{CD} 69^{+}$regions after deducting background staining (isotypic $m A b$ ). For the last panel, region $A$ represents all $C D 69-$ positive cells, and region $B$ represents cells with high CD69 intensity. (b) Sorted EphB6 ${ }^{+}$(solid line) and EphB6- (dotted line) cells were cultured in wells coated with anti-CD3 and anti-CD28, as described above, for an additional $1-3$ days. Their thymidine uptake was measure for the last 8 hours of culture. 
a

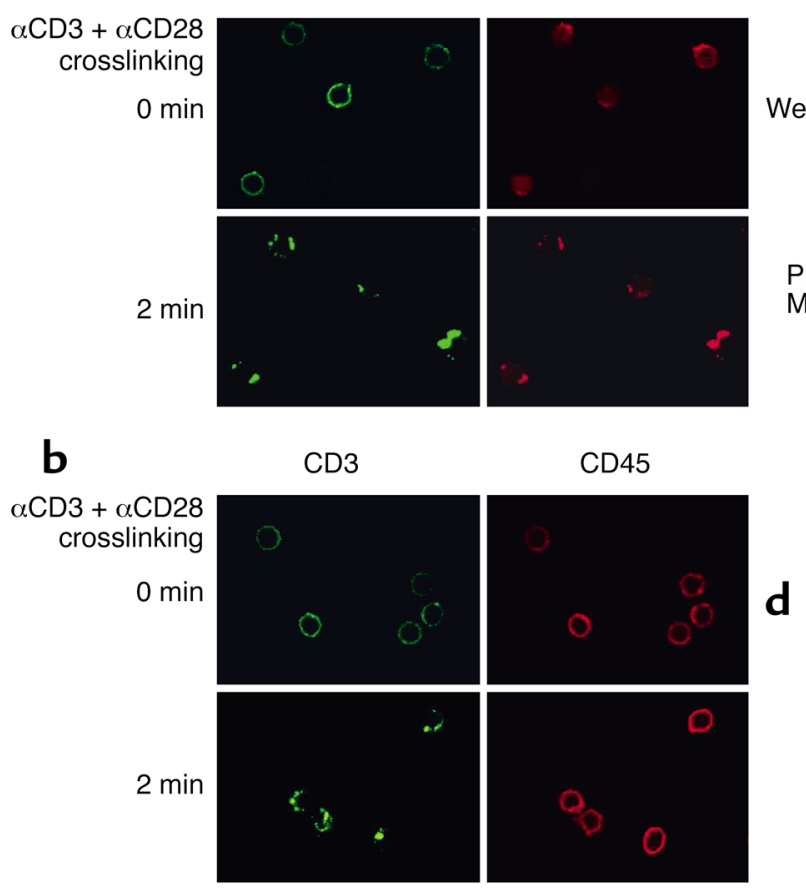

C

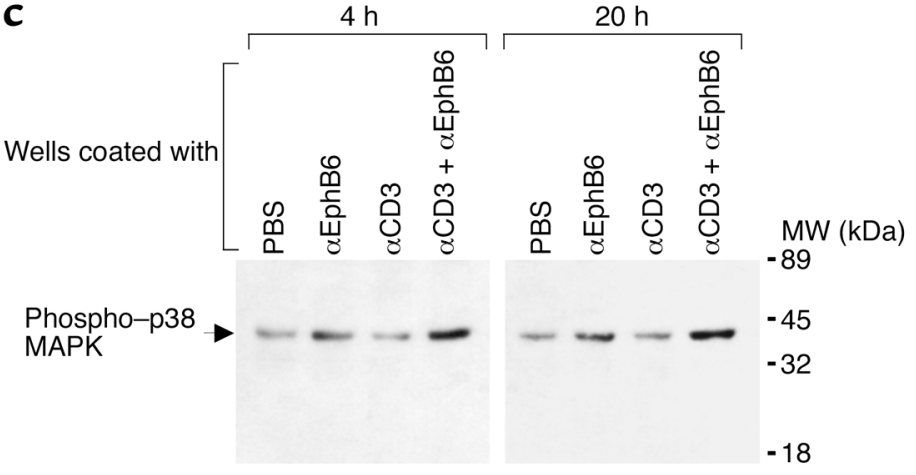

WB: anti-phospho-p38 MAP Kinase

p38 MAPK

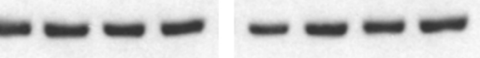

Reprobe: anti-p38 MAP Kinase

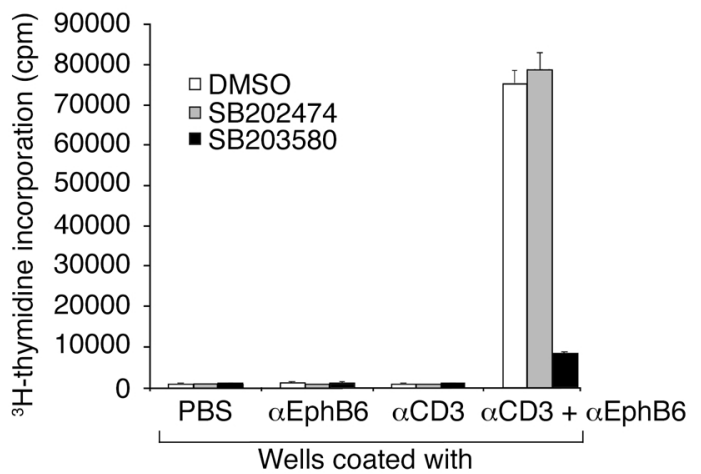

\section{Figure 5}

EphB6 signaling. (a) Rapid colocalization of EphB6 with TCR caps after anti-CD3 and anti-CD28 crosslinking. All experiments were conducted more than three times and were reproducible. Representative results are shown. MACS-purified T cells were cultured for 48 hours in medium and then incubated with Alexa Fluor 488-labeled anti-CD3 mAb and anti-CD28 mAb on ice for 40 minutes, followed by rabbit anti-mouse IgG crosslinking at $37^{\circ} \mathrm{C}$ for 2 minutes. Cells were immediately fixed with $4 \%$ paraformaldehyde and stained with biotinylated anti-EphB6 mAb followed by Alexa Fluor 594-conjugated streptavidin. The cells were then examined by confocal microscopy for CD3 (green) and EphB6 (red) localization. (b) CD45 does not translocate to TCR caps after anti-CD3 and anti-CD28 crosslinking. The cells were crosslinked and stained as in Figure 5a, except that biotinylated anti-CD45 mAb was used in place of biotinylated anti-EphB6 mAb. (c) Immunoblotting of p38 MAPK and tyrosine phosphorylated p38 MAPK (phospho-p38 MAPK). MACS-purified T cells were added to wells coated with anti-EphB6 $(4 \mu \mathrm{g} / \mathrm{ml})$ and/or anti-CD3 $(0.2 \mu \mathrm{g} / \mathrm{ml})$. The cells were harvested after 4 hours or 20 hours, as indicated, and analyzed by immunoblotting. Signals of 38 MAPK and phospho-p38 MAPK on the same membrane are indicated by arrows. (d) Effects of the p38 MAPK-specific inhibitor on EphB6-enhanced T cell proliferation. MACS-purified T cells were preincubated for 1 hour in complete culture medium containing the p38 MAPK-specific inhibitor SB203580 (10 $\mu \mathrm{M})$, its nonfunctional structural analogue SB202474 (10 $\mu \mathrm{M})$, or vehicle (DMSO; $0.1 \%)$. The cells were then transferred to wells coated with the anti-EphB6 mAb 4F12 (4 $\mu \mathrm{g} / \mathrm{ml})$, the anti-CD3 mAb OKT3 $(0.2 \mu \mathrm{g} / \mathrm{ml})$, or both, and cultured for 72 hours. ${ }^{3} \mathrm{H}$-thymidine was added to the culture for the last 8 hours, and thymidine uptake by the cells was measured. MW, molecular weight; WB, Western blot.

PHA-stimulated T cells have repressed EphB6 mRNA levels (15), and can no longer express EphB6 (see Figure 1b). The need for EphB6 in costimulation and the suppression of EphB6 expression after mitogen stimulation seem paradoxical at first glance. However, this becomes logical if EphB6 signaling is needed only in the first several minutes or hours of $\mathrm{T}$ cell activation. Indeed, when T cells were stimulated by TCR and CD28 co-crosslinking, which mimicked in vivo $\mathrm{T}$ cell response to antigen-presenting cells, EphB6 rapidly congregated (within 2 minutes) and migrated to the TCR caps. This provides a morphological basis for EphB6 to enhance TCR signaling by physically engaging the EphB6 signaling platform with that of TCR. As this event occurred almost immediately after TCR crosslinking, it is reasonable to assume that EphB6 signaling is employed very early in the $\mathrm{T}$ cell activation process, but is unnecessary or even harmful after $\mathrm{T}$ cells are activated. This justifies the need for subsequent EphB6 downregulation.

It is worth mentioning that EphB6 costimulation was effective in the pure $T$ cell system as shown in Figure 4 , suggesting that it can happen between $T$ cells. This finding was not an artifact due to use of antiEphB6 mAb during cell sorting, nor was this due to Eph expression being merely a sign of, and not a cause for, better $\mathrm{T}$ cell responsiveness. We know this because purified T cells from $E p h B 6$ gene knockout mice also had a significantly compromised response to CD3 and CD28 stimulation compared with that from wild-type 
littermates, while the cellularity and immune cell composition in the spleen and thymus of the knockout and wild-type littermates were comparable (unpublished observations). It is well known that $\mathrm{T}$ cells need to reach a certain density in culture to optimally respond to TCR stimulation; this is often attributed to the need for cytokines in culture. Without discounting the importance of cytokines, costimulation between $\mathrm{T}$ cells via membrane-bound Eph's and ephrins can well explain the cell density requirement for their activation. It can also explain why in vivo $T$ cell responses occur in densely populated $\mathrm{T}$ cell regions. Such $\mathrm{T}$ cell- $\mathrm{T}$ cell costimulation certainly does not exclude the possible coexistence of $\mathrm{T}$ cell-antigen-presenting cell costimulation involving Eph's and ephrins.

Costimulation by EphB6 led to secretion of many lymphokines, but IL-2 was notably absent. We wondered whether there was a lack of IL-2 secretion because EphB6 activated only $\mathrm{CD}^{+}$cells or recently activated T cells, which are low IL-2 producers. This did not seem to be the case, because $\mathrm{CD}^{+}$cells and naive $\mathrm{CD}^{2} 5 \mathrm{RO}^{-}$ $\mathrm{T}$ cells also vigorously proliferated in response to EphB6 costimulation. It is quite possible that other lymphokines compensate for the function of IL-2 in supporting $\mathrm{T}$ cell proliferation.

The pattern of lymphokine secretion stimulated by EphB6 costimulation is distinctive from that of CD28 costimulation, besides the lack of IL-2 production in the former and abundant IL-2 production in the latter. When pure $\mathrm{T}$ cells were stimulated by anti-CD3 and anti-CD28, both IFN- $\gamma$ and IL-4 were produced (21). However, EphB6 costimulation led to no IL-4 secretion, while IFN- $\gamma$ production was intact. This raised the possibility that ephB6 costimulation preferably drives $\mathrm{T}$ cells to differentiate into a Th1 phenotype. On the other hand, EphB6 costimulation also enhances TGF- $\beta$ secretion, which is reportedly associated with conditions favoring Th2 phenotype differentiation (22). Therefore, EphB6 does not seem to provide classical costimulation like CD28, and might serve a different purpose. Indeed, we recently came to the understanding that there are multiple costimulating pathways with different consequences. Some examples: signals through inducible costimulator (ICOS) affect activated T cells, and this pathway enhances IL-10 but not IL-2 production (23); costimulation via signaling lymphocyte activation molecule (SLAM) enhances IFN- $\gamma$ production and directs memory $T$ cells toward Th0/Th1 responses (24); crosslinking of 4-1BB preferentially promotes $\mathrm{CD}^{+}$cell proliferation (25); and ligation of OX40 increases Th2 responses (26). The precise role of EphB6 costimulation on Th1/Th2 differentiation and on other aspects of $\mathrm{T}$ cell biology apparently needs to be further elucidated; such study is currently in progress.

A recent study showed that $\mathrm{T}$ cell activation depends on cytoskeletal reorganization (27), which facilitates the formation of focal contact sites between $T$ cells and antigen-presenting cells (28). The known function of
Eph kinases in the CNS is spatial patterning and cell positioning. Such a function logically depends on altering cytoskeletal structures. Indeed, EphA5/REK7 activation disrupts cytoskeletal organization in the growth cone of neurons (29). Quite likely, EphB6 costimulation in T cells is related to the eventual effect of EphB6 on the cytoskeleton, as with other members of Eph kinases in the CNS. Small GTP-binding proteins such as Rac-1, Cdc42, and Rho transduce signals the cytoskeleton and are implicated in the effect of other Eph kinases $(30,31)$. We demonstrated that p38 MAPK, which is a downstream signaling molecule in the Rac-1/Cdc42/Rho/Pak-1 signaling pathway (32, 33 ), is activated after EphB6 and CD3 co-crosslinking. These are circumstantial pieces of evidence suggesting the involvement of the cytoskeleton in EphB6 costimulation. Recently, it has been reported that EphB2 activation results in inhibition of $\mathrm{p} 44 / \mathrm{p} 42 \mathrm{MAPK}$ in neuronal cells (34), and that EphA activation leads to inhibition of this kinase in several cell lines of endothelial and epithelial origin (35). Obviously, these reports deal with cell types, Eph kinases, and MAPKs that are different from those in our study. The consequence of Eph activation in other cells is different from that in $\mathrm{T}$ cells as well. In neuronal cells, endothelial cells, and epithelial cells, Eph activation does not induce cell proliferation, while in $\mathrm{T}$ cells, it does. Further comparative studies on EphB6 signaling in immune and nonimmune cells are warranted.

Our study on the function of Eph kinases has revealed previously unknown functions of this largest family of receptor tyrosine kinases in the immune system. We believe that some of these molecules are important in $\mathrm{T}$ cell activation, differentiation, and homeostasis, based on our current findings.

\section{Acknowledgments}

This work was supported by grants from the Canadian Institutes of Health Research (CIHR; MT-15673, P-57321, and MOP-57697); the CIHR/Canadian Blood Service Partnership Program; the Heart and Stroke Foundation of Quebec; the Kidney Foundation of Canada; the Roche Organ Transplantation Research Foundation, Switzerland (ROTRF; 474950960); the Juvenile Diabetes Research Foundation, USA (5-2001540); and the J-Louis Levesque Foundation (to J. Wu). $\mathrm{J}$. Wu is a National Researcher of the Quebec Health Research Foundation. The authors acknowledge the editorial assistance of Ovid Da Silva, Redacteur, Research Support Office, Research Center, CHUMHotel-Dieu, Montreal, Canada.

1. Abraham, R.T., Karnitz, L.M., Secrist, J.P., and Leibson, P.L. 1992. Signal transduction through the T-cell antigen receptor. Trends Biochem. Sci. 17:434-438.

2. 1997. Unified nomenclature for Eph family receptors and their ligands, the ephrins. Eph Nomenclature Committee. Cell. 90:403-404.

3. Holland, S.J., et al. 1996. Bidirectional signaling through the Eph-family receptor Nuk and its transmembrane ligand. Nature. 383:722-725.

4. Flanagan, J.G., and Vanderhaeghen, P. 1998. The ephrins and Eph receptors in neural development. Annu. Rev. Neurosci. 21:309-345.

5.Xu, Q., and Wilkinson, D.G. 1997. Eph-related receptors and their 
ligands: mediators of contact dependent cell interactions. J. Mol. Med. 75:576-586

6. Leighton, P.A., et al. 2001.Defining brain wiring patterns and mechanisms through gene trapping in mice. Nature. 410:174-179.

7. Kullander, K., et al. 2001. Kinase-dependent and kinase-independent functions of EphA4 receptors in major axon tract formation in vivo. Neuron. 29:73-84.

8. Holmberg, J., Clarke, D.L., and Frisen, J. 2000. Regulation of repulsion versus adhesion by different splice forms of an Eph receptor. Nature. 408:203-206.

9. Gerlai, R. 2001. Eph receptors and neural plasticity. Nat. Rev. Neurosci. 2:205-209.

10. Wang, H.U., Chen, Z.F., and Anderson, D.J. 1998. Molecular distinction and angiogenic interaction between embryonic arteries and veins revealed by ephrin-B2 and its receptor Eph-B4. Cell. 93:741-753.

11. Lackmann, M., et al. 1996. Purification of a ligand for the EPH-like receptor HEK using a biosensor-based affinity detection approach. Proc. Natl. Acad. Sci. USA. 93:2523-2527.

12. Andres, A.C., et al. 1994. Expression of two novel eph-related receptor protein tyrosine kinases in mammary gland development and carcinogenesis. Oncogene. 9:1461-1467.

13. Ciossek, T., Lerch, M.M., and Ullrich, A. 1995. Cloning, characterization, and differential expression of MDK2 and MDK5, two novel receptor tyrosine kinases of the eck/eph family. Oncogene. 11:2085-2095.

14. Gurniak, G.B., and Berg, L.J. 1996. A new member of the Eph family of receptors that lacks protein tyrosine kinase activity. Oncogene. 13:777-786

15. Luo, L., Wan, X., Wu, Y., and Wu, J. 2001. Cross-linking of EphB6 resulting in signal transduction and apoptosis in Jurkat cells. J. Immunol. 167:1362-1370.

16. Shimoyama, M., et al. 2000. T-cell-specific expression of kinase-defective Eph-family receptor protein, EphB6 in normal as well as transformed hematopoietic cells. Growth Factors. 18:63-78.

17. Matsuoka, H., et al. 1997. Expression of a kinase-defective Eph-like receptor in the normal human brain. Biochem. Biophys. Res. Commun. 225:487-492.

18. Luo, H., et al. 1992. Inhibition of in vitro immunoglobulin production by rapamycin. Transplantation. 53:1071-1076.

19. Chen, H., et al. 1996. Impaired signaling in alloantigen-specific $\mathrm{CD}^{+} \mathrm{T}$ cells tolerized in vivo. Employing a model of $\mathrm{L}^{\mathrm{d}}$-specific TCR transgenic mice transplanted with allogenic hearts under the cover of a short-term rapamycin treatment. J. Immunol. 157:4297-4308.

20. Munthe, E., et al. 2000. Ephrin-B2 is a candidate ligand for the Eph receptor EphB6. FEBS Lett. 466:169-174.

21. King, C.L., Stupi, R.J., Craighead, N., June, C.H., and Thyphronitis, G.
1995. CD28 activation promotes Th2 subset differentiation by human CD4+ cells. Eur. J. Immunol. 25:587-595.

22. Seder, R.A., et al. 1998. Factors involved in the differentiation of TGFbeta-producing cells from naive CD4+ T cells: IL-4 and IFN-gamma have opposing effects, while TGF-beta positively regulates its own production. J. Immunol. 160:5719-5728.

23. Hutloff, A., et al. 1999. ICOS is an inducible T-cell co-stimulator structurally and functionally related to CD28. Nature. 397:263-266.

24. Aversa, G., Chang, C.C., Carballido, J.M., Cocks, B.G., and de Vries, J.E. 1997. Engagement of the signaling lymphocytic activation molecule (SLAM) on activated T cells results in IL-2-independent, cyclosporin Asensitive $\mathrm{T}$ cell proliferation and IFN-gamma production. J. Immunol. 158:4036-4044

25. Shuford, W.W., et al. 1997. 4-1BB costimulatory signals preferentially induce CD8+ $\mathrm{T}$ cell proliferation and lead to the amplification in vivo of cytotoxic T cell responses. J. Exp. Med. 186:47-55.

26. Flynn, S., Toellner, K.M., Raykundalia, C., Goodall, M., and Lane, P. 1988. CD4 $\mathrm{T}$ cell cytokine differentiation: the B cell activation molecule, OX40 ligand, instructs CD4 T cells to express interleukin 4 and upregulates expression of the chemokine receptor, Blr-1. J. Exp. Med. 188:297-304.

27. Penninger, J.M., and Crabtree, G.R. 1999. The actin cytoskeleton and lymphocyte activation. Cell. 96:9-12.

28. Monks, C.R., Freiberg, B.A., Kupfer, H., Sciaky, N., and Kupfer, A. 1998 Three-dimensional segregation of supramolecular activation clusters in T cells. Nature. 395:82-86

29. Meima, L., et al. 1997. AL-1-induced growth cone collapse of rat cortical neurons is correlated with REK7 expression and rearrangement of the actin cytoskeleton. Eur. J. Neurosci. 9:177-188.

30. Shamah, S.M., et al. 2001. EphA receptors regulate growth cone dynamics through the novel guanine nucleotide exchange factor ephexin. Cell. 105:233-244.

31. Holland, S.J., et al. 1997. Juxtamembrane tyrosine residues couple the Eph family receptor EphB2/Nuk to specific SH2 domain proteins in neuronal cells. EMBO J. 16:3877-3888.

32. Cano, E., and Mahadevan, L.C. 1995. Parallel signal processing among mammalian MAPKs. Trends Biochem. Sci. 20:117-122.

33. Salojin, K.V., Zhang, J., and Delovitch, T.L. 1999. TCR and CD28 are coupled via ZAP-70 to the activation of the Vav/Rac-1-/PAK-1/p38 MAPK signaling pathway. J. Immunol. 163:844-853.

34. Elowe, S., Holland, S.J., Kulkarni, S., and Pawson, T. 2001. Downregulation of the Ras-mitogen-activated protein kinase pathway by the EphB2 receptor tyrosine kinase is required for ephrin-induced neurite retraction. Mol. Cell. Biol. 21:7429-7441.

35. Miao, H., et al. 2001. Activation of EphA receptor tyrosine kinase inhibits the Ras/MAPK pathway. Nat. Cell Biol. 3:527-530. 